\title{
eJRIEPS
}

Ejournal de la recherche sur l'intervention en éducation physique et sport

43 | 2018

Varia

\section{Analyse didactique clinique de pratiques déclarées par des enseignants d'EPS : un déjà-là en tension dans l'enseignement du football en Tunisie}

Maher Guerchi, Hanene Lengliz et Denis Loizon

\section{(2) OpenEdition}

Journals

Édition électronique

URL : http://journals.openedition.org/ejrieps/467

DOI : 10.4000 /ejrieps.467

ISSN : 2105-0821

Éditeur

ELLIADD

Référence électronique

Maher Guerchi, Hanene Lengliz et Denis Loizon, « Analyse didactique clinique de pratiques déclarées par des enseignants d'EPS : un déjà-là en tension dans l'enseignement du football en Tunisie », eJRIEPS [En ligne], 43 | 2018, mis en ligne le 01 janvier 2018, consulté le 01 août 2019. URL : http:// journals.openedition.org/ejrieps/467; DOI : 10.4000/ejrieps.467

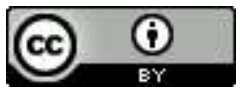

La revue eJRIEPS est mise à disposition selon les termes de la Creative Commons Attribution 4.0 International License. 
eJRIEPS 43 janvier 2018

Analyse didactique clinique de pratiques déclarées par des enseignants

d'EPS : un déjà-là en tension dans l'enseignement du football en Tunisie

Guerchi Maher ${ }^{\star}$, Lengliz Hanene ${ }^{\star \star}$, Loizon Denis ${ }^{* \star *} \&$ Marie-France Carnus ${ }^{\star * * *}$

*UMR EFTS MA 122 (EDiC), ISSEPS KEF, Tunisie

**UMR EFTS MA 122 (EDiC), ISSEP Ksar Daid Tunis, Tunisie

${ }^{* * * S P M S}$ EA 4180 (EDiC), Université de Bourgogne, France

****UMR EFTS MA 122 (EDiC), Université de Toulouse Jean Jaurés, France

Résumé

Nous présentons ici une étude exploratoire réalisée dans le cadre d'une thèse portant sur l'analyse didactique clinique des pratiques enseignantes de quatre enseignants d'EPS tunisiens dans l'activité football. A travers un questionnaire où 167 enseignants ont répondu nous mettons en évidence des tensions et des contradictions au sein d'un déjà-là décisionnel composé de trois composantes conceptuelle, intentionnelle et expérientielle. En amont du processus décisionnel, ce déjà-là agit comme "filtre de l'action didactique " d'où émergent les savoirs à enseigner au cours d'un processus de transposition didactique influencé par des facteurs culturels, institutionnels et personnels. En ouvrant la voie à d'autres réseaux de signification ces travaux mettent en évidence la part de subjectivité des enseignants d'EPS dans le traitement de l'activité football déclaré par les enseignants, apportent un nouvel éclairage à la complexité du fonctionnement didactique ainsi qu'une autre compréhension de la difficulté à enseigner le football en milieu scolaire.

Mots clés : enjeux de savoirs, football, pratiques déclarées, transposition didactique, didactique clinique de l'EPS, déjà-là décisionnel

\section{Introduction}

Certains travaux en didactique clinique de l'EPS ont révélé les écarts parfois considérables entre les conceptions, croyances et idéaux qui animent les enseignants, les intentions qui les orientent et leurs choix didactiques effectifs 


\section{eJRIEPS 43 janvier 2018}

lorsqu'ils sont confrontés à l'épreuve de la transmission - appropriation (Carnus, 2003). Ces écarts sont de nature à s'intensifier lorsque l'enseignant se trouve dans des situations problématiques marquées par la contingence dont les origines sont multiples : institutionnelle, contextuelle et surtout personnelle. L'analyse de ces écarts rend compte des phénomènes transpositifs des savoirs en jeu de leur sélection à leur transmission par l'enseignant jusqu'à leur appropriation par l'élève.

Ainsi, centrées sur le suivi des enjeux de savoir dans le cadre d'une relation ternaire, les recherches en didactique clinique questionnent l'influence de la trajectoire personnelle des enseignants sur leurs pratiques professionnelles. Ouvrant la voie à une autre lecture du fonctionnement didactique, les travaux de l'EDiC ${ }^{1}$ montrent qu'au-delà des difficultés et des contraintes externes liées aux conditions de la pratique d'enseignement en EPS, de nombreux facteurs internes interviennent parfois à l'insu de l'enseignant et semblent avoir un rôle déterminant dans ses prises de décision. Ces influences internes - produits de l'histoire personnelle des enseignants - orientent leurs choix avant et pendant l'action didactique.

Prenant appui sur une théorie du sujet pris dans le didactique ${ }^{2}$ (Carnus, Terrisse, 2013), l'EDiC a élaboré une stratégie de recherche in situ et « au cas par cas » (Terrisse, 2000) qui rend compte des pratiques enseignantes à la croisée de la didactique de l'EPS et de la clinique psychanalytique. Cette stratégie s'accompagne de la construction d'un appareillage conceptuel et méthodologique permettant de décrire et de comprendre l'étude du processus de transmission - appropriation des enjeux de savoir en même temps que le caractère singulier de cette transmissionappropriation (Terrisse, Carnus, Loizon, 2010).

Dans sa thèse portant sur l'enseignement de la gymnastique, Carnus a repéré à travers un questionnaire l'existence d'un « déjà-là décisionnel » chez les enseignants d'EPS. Marquée par les tensions et les contradictions, cette "partie cachée du processus décisionnel de l'enseignant lui fournit, à moments donnés, des motifs et mobiles possibles dans le cadre de son activité professionnelle " Carnus (2001, p. 376). Notre intention est de transposer la démarche et le concept dans une autre APSA support et dans un autre contexte culturel pour faire émerger un déjà-là décisionnel chez des enseignants d'EPS. Aussi, cet article rend compte d'une étape

\footnotetext{
${ }^{1}$ Equipe de recherche en Didactique Clinique de l'EPS

${ }^{2}$ Le sujet est singulier, assujetti et divisé dans et par son inconscient (Carnus, 2007)
} 


\section{eJRIEPS 43 janvier 2018}

exploratoire d'un travail de thèse portant sur l'analyse didactique clinique de pratiques enseignantes d'EPS en Tunisie. Dans la continuité des travaux de Touboul (2010), notre projet à terme sera de rendre compte chez quatre enseignants aux profils contrastés du poids de l'expertise ${ }^{3}$ et du sexe dans l'enseignement d'une activité genrée très médiatisée et privilégiée en Tunisie : le football. A ce stade de notre recherche, nous rendons compte des pratiques déclarées par 167 enseignants d'EPS tunisiens à travers l'étude des réponses à un questionnaire qui tente de repérer certains phénomènes relevant d'un " déjà-là sous tension » influençant les enjeux de savoir dans l'activité football en arrière-plan du système didactique.

\section{Quels savoirs visés en football à l'école ? Une transposition sous influence}

L'enseignement du football dans les établissements scolaires passe en amont par une formalisation des savoirs à enseigner que Chevallard (1992) nomme la « mise en texte du savoir " de nature à prendre en compte la dimension des apprentissages scolaires, la singularité de l'enseignant et des apprenants en même temps que la particularité des contextes d'enseignement. Ce processus de transposition didactique fait émerger un ensemble de transformations, de disparitions, de créations, et - in fine - des écarts entre la pratique sociale de référence et la pratique scolaire.

En didactique clinique la transposition didactique a été revisitée sous l'angle de trois sources d'influences : socioculturelle, institutionnelle et personnelle (Carnus, 2009 ; Heuser, 2009). Les deux premières renvoient à des facteurs externes en lien avec les pratiques sociales de référence - ici le football de haut-niveau - et les contraintes relatives à l'institution scolaire - notamment les choix fait par l'école en matière de programme et d'organisation de l'enseignement. La troisième source d'influences touche aux facteurs internes propres aux sujets engagés dans la relation didactique, leur singularité, leur histoire, leur déjà-là. Ces trois sources d'influences interagissent à chaque étape de la transposition didactique : du savoir à enseigner, au savoir enseigné jusqu'au savoir appris par l'élève (Heuser, 2009). Nous nous focalisons ici sur la première étape relative à l'identification des savoirs à enseigner.

1. 1. Les influences socioculturelles et institutionnelles

Certains travaux s'intéressent à l'identification des savoirs transmis aux footballeurs

${ }^{3}$ En termes de spécialisation 


\section{eJRIEPS 43 janvier 2018}

de haut niveau. Par exemple, Cazorla et Farhi (1998) s'adressent aux entraîneurs nationaux et internationaux en leur demandant : " quelles sont les qualités indispensables au footballeur de haut niveau? ?. Parmi les réponses les plus citées, on trouve : les qualités cognitives et mentales (capacités d'adaptation, sens tactique, agressivité) ; les qualités techniques ; les qualités physiques et physiologiques (bonne constitution, vitesse, puissance). Leurs analyses mettent en avant qu'en football « les contenus d'entraînement s'appuient de plus en plus sur les exigences imposées par le match et sur le niveau des capacités du joueur » (Cazorla, Farhi, 1998). Dans ce contexte, les experts convoquent différents registres de savoir : des savoir-être (adaptation, agressivité et mental) et des savoirs techniques et tactiques en rapport avec la mobilisation de ressources cognitives et physiologiques - ces dernières étant déterminantes dans la réussite en football.

Le football occupe aujourd'hui une place importante et privilégiée en Tunisie. L'école, dans les missions culturelles et éducatives qui lui sont assignées, notamment « apprendre à faire et à comprendre " ne peut passer à côté de ces pratiques sociales très pratiquées et motivantes pour une grande partie des élèves. En tant qu'activité physique et sportive moyen de l'EPS, le football, programmé à l'école, contribue à l'acquisition de nouvelles conduites et habiletés motrices spécifiques en même temps qu'au développement des capacités physiques, des connaissances et des savoirs nécessaires à la gestion de la vie physique.

Entre référence culturelle et référence scolaire, quels savoirs en football sont transposables à l'école ? Au-delà de l'influence de ces facteurs externes quelle part prennent les influences personnelles en lien avec l'histoire et la singularité du sujet enseignant dans le processus de transposition didactique ?

1. 2. Les influences personnelles : le déjà-là décisionnel

Nous nous centrons ici sur les facteurs internes propres au sujet enseignant influençant les enjeux de savoir. Ces facteurs internes renvoient à ce que Carnus appelle un déjà-là décisionnel. Ce déterminant personnel de la décision enseignante (Loizon, Carnus, 2012) est composé de trois instances en tension : le déjà-là conceptuel, le déjà-là intentionnel et le déjà-là expérientiel (Carnus, 2001).

Le déjà-la conceptuel s'appuie sur la notion de conceptions en rapport avec le savoir en jeu. Pour Giordan et De Vecchi (1987), la conception est un élément moteur entrant dans la construction d'un savoir. Cette notion relève du domaine 


\section{eJRIEPS 43 janvier 2018}

cognitif et permet de donner du sens aux enjeux de savoirs. Le déjà-là conceptuel est construit par le sujet au cours de son histoire et de sa trajectoire professionnelle. Enraciné dans la sphère privée, il est également influencé par ce qui relève de la sphère publique : institutionnelle - notamment à travers les programmes d'enseignement - et socioculturelle au regard de l'essence de la pratique sociale de référence et de sa place dans une culture donnée. Le déjà-là conceptuel constitue le pôle cognitif du déjà-là décisionnel (Carnus, 2001, 2002).

Le déjà là intentionnel fait appel à la notion d'intention qui se définit comme " la tension vers un but, la direction vers un objet " Carnus (2001). Le même auteur signale la difficulté d'accéder à une telle dimension en situation d'enseignementapprentissage du fait de la pluralité et la multiplicité des intentions organisées en réseau complexe au sein duquel se trouvent les intentions didactiques relatives aux savoirs visés par l'enseignant. Ces savoirs à enseigner sont les produits de la transposition didactique interne (Chevallard, 1985 ; Portugais, 1999). Tout comme le déjà-là conceptuel, le déjà-là intentionnel est sous la triple influence sociale, institutionnelle et personnelle. Loizon $(2004,2009)$ distingue le « déjà-là intentionnel spécifique » et le « déjà-là intentionnel général ». Le premier est relatif aux intentions didactiques en lien avec l'APSA support de l'enseignement, le second est relatif aux intentions éducatives portant sur des aspects méthodologiques et/ou sociaux et qui s'étendent souvent sur une période plus longue que la leçon ou le cycle d'EPS. Ainsi, au cœur du déjà-là intentionnel agissant comme " filtre de l'action didactique " (Loizon, 2004), se trouve le savoir à enseigner, produit sans cesse remanié d'une épistémologie personnelle et professionnelle au carrefour du privé et du public. Le déjà-là intentionnel constitue le pôle conatif du déjà-là décisionnel (Carnus, 2001).

Le déjà-là expérientiel fait référence à l'expérience personnelle et professionnelle de l'enseignant en tant que vécu qui a laissé au cours son histoire des traces plus ou moins indélébiles et pas toujours conscientisées voir conscientisables. II s'agit d'un ensemble de savoirs d'expérience dans différents contextes et institutions qui constitue pour l'enseignant un répertoire d'éprouvés plus ou moins agréables que le chercheur didacticien clinique cherche dans un premier temps à investiguer à travers le discours de l'enseignant pour tenter dans un second temps d'en inférer l'influence sur ses pratiques professionnelles effectives ${ }^{4}$.

\footnotetext{
${ }^{4}$ En didactique clinique, ce second temps est celui de l'épreuve.
} 


\section{eJRIEPS 43 janvier 2018}

La figure 1 schématise le déjà-là décisionnel et ses trois pôles conceptuel, intentionnel et expérientiel agissant comme des filtres personnels de l'action didactique à l'origine des choix des enseignants. Les travaux en didactique clinique de l'EPS mettent en avant les tensions et contradictions à l'œuvre au sein du déjà-là décisionnel.

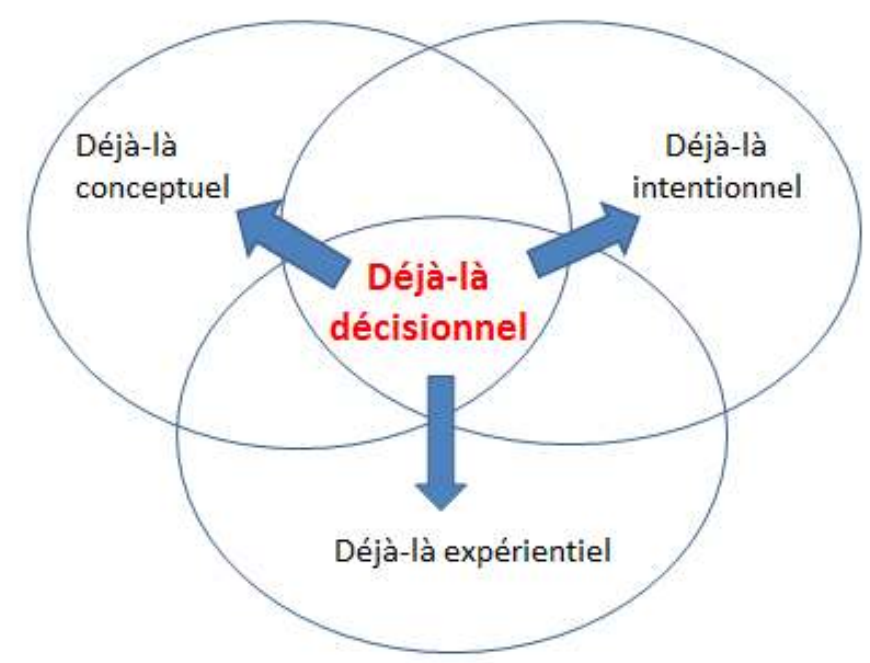

Figure 1. Les trois pôles du déjà-la décisionnel

Pour accéder aux différents volets du déjà-là décisionnel et repérer ses tensions structurelles constitutives entre cognition, conation et expérience agissant en arrièreplan des décisions, le chercheur peut utiliser un questionnaire et des entretiens préliminaires réalisés en amont de l'observation des pratiques effectives.

A cette étape préalable de notre recherche nous cherchons à repérer, entre influences culturelles, institutionnelles et personnelles, les savoirs relatifs à l'enseignement du football que les enseignants déclarent enseigner. A travers l'émergence d'un déjà-là décisionnel chez les enseignants d'EPS Tunisien, notre intention est de rendre compte de leurs pratiques déclarées lorsqu'il s'agit d'enseigner un cycle de football.

\section{Méthodologie}

Pour cette étude exploratoire nous avons recours à un questionnaire composé de 26 questions conçues et regroupées de manière qualitative autour de trois thématiques : 


\section{eJRIEPS 43 janvier 2018}

les conceptions et références professionnelles les plus citées par les enseignants d'EPS (1) ; les intentions spécifiques des enseignants en rapport avec les savoirs visés en football et les intentions générales en lien avec des finalités éducatives (2) ; les pratiques usuelles en relation avec la manipulation de certaines variables didactiques $(3)^{5}$. Les réponses sont traitées de manière quantitative. Les questions ouvertes donnent lieu à une catégorisation des réponses suite à une analyse sémantique de contenu (Bardin, 1998). Nous avons recours à des outils classiques de statistiques descriptives (pourcentages et histogrammes) pour traiter et présenter les résultats. Au cas échéant, un test de chi2 permet de repérer d'éventuelles liaisons au regard des caractéristiques des répondants et notamment le sexe et l'expertise ${ }^{6}$.

Ainsi, la nature des questions et l'analyse des réponses nous permet de dresser à titre exploratoire un premier état des lieux d'un déjà-là décisionnel chez les enseignants d'EPS dans ses trois dimensions : conceptuelle, intentionnelle et expérientielle ${ }^{7}$.

167 enseignants d'EPS tunisiens ont répondu à ce questionnaire dont 58 femmes (35\%) et 109 hommes (65\%). Leur ancienneté dans le métier varie de 0 à plus de 26 ans $^{8}$. 49 sont spécialistes (29\%) et 118 non spécialistes (71\%). Enfin, 23 exercent dans une école primaire (14\%), 85 en collège (51\%) et 59 en lycée (35\%).

\section{Quelques résultats}

Nous sélectionnons ici certains résultats qui nous apparaissent les plus significatifs au regard de notre questionnement. Nous organisons la présentation de ces résultats en fonction des trois déjà-là présentés précédemment.

\footnotetext{
${ }^{5}$ Le dispositif d'analyse des pratiques effectives s'inscrit dans une méthodologie d'ingénierie didactique permettant de créer un contexte d'observation favorable à l'étude du fonctionnement didactique (Artigue, 1990 ; Amade-Escot \& Léziart, 1996). Les réponses à certaines questions nous permettent également de repérer d'éventuels écarts entre les options de l'ingénierie et les pratiques usuelles des quatre enseignants qui ont accepté de collaborer à notre étude.

${ }^{6}$ Ces deux critères (sexe et expertise) différencient les quatre enseignants associés à la recherche. Deux femmes dont une spécialiste et l'autre non spécialiste et deux hommes dont un spécialiste et l'autre non spécialiste. Tous les quatre sont débutants.

${ }_{7}^{7}$ Cet état des lieux indiquera certaines tendances du déjà-là décisionnel des enseignants d'EPS interrogés et permettra

de situer, de nuancer et de pondérer l'étude des déjà-là décisionnels des quatre enseignants associés à la recherche. Cette étude se fera à partir d'une analyse qualitative d'entretiens que nous effectuerons en amont de l'observation in situ des pratiques.

${ }^{8}$ La totalité des enseignants sont répartis en tranche d'ancienneté de la façon suivante : 41 de 1 à 3 ans (dont nos quatre collaborateurs), 29 de 4 à 10 ans, 31 de 11 à 15 ans, 33 de 16 à 20 ans, et 33 ont plus de 20 ans d'ancienneté.
} 


\section{eJRIEPS 43 janvier 2018}

\section{1. Le déjà-là conceptuel}

Nous analysons ici les réponses à deux questions : quelle est votre définition du football

(1) ? Que pensez-vous du football à l'école (2) ? La première renvoie aux conceptions de la pratique sociale de référence ; la seconde à celles de la pratique scolaire. Les écarts constatés dans les réponses à ces questions rendent compte du processus transpositif à l'œuvre chez les enseignants d'EPS tunisiens interrogés.

Pour mettre en relief les conceptions dominantes relatives à la pratique sociale de référence, nous avons découpé les réponses à cette question en unités de sens et les avons regroupé a posteriori en quatre catégories : sport d'équipe ; activité d'affrontement ; activité à connotation masculine ; activité d'opposition et de coopération. La première unité de sens qui apparait dans chaque réponse renvoie à ce que nous considérons comme la conception dominante du répondant. Le graphique 1 représente la distribution en nombre et en pourcentage des conceptions dominantes relatives au football en tant que pratique sociale de référence.

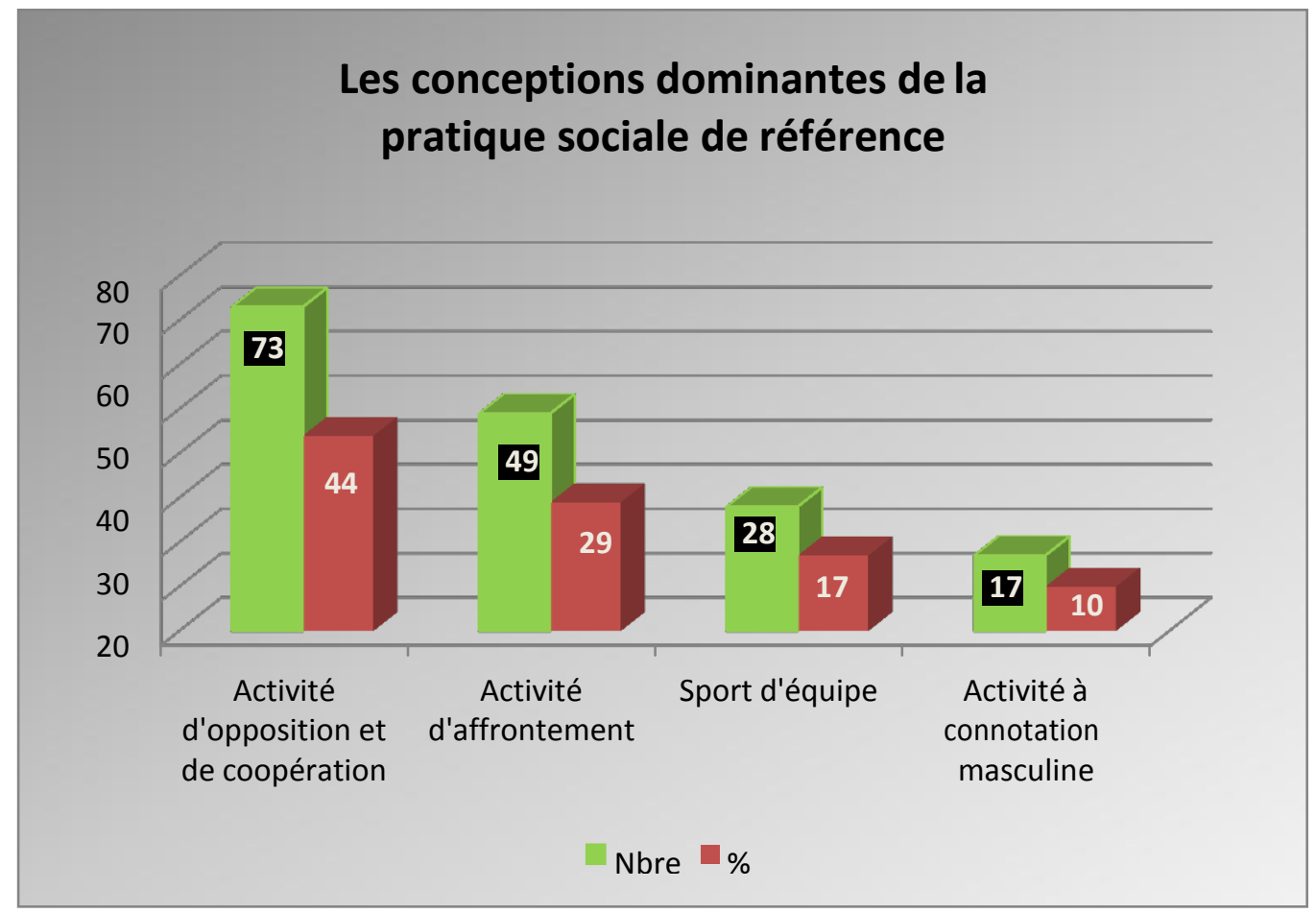

Graphique 1. Les conceptions dominantes du football.

Pour $44 \%$ des enseignants ayant répondu au questionnaire le football se présente en premier lieu comme une activité d'opposition et de coopération. Pour $29 \%$ d'entre eux le football est d'abord un affrontement. $17 \%$ des réponses mettent en avant la 


\section{eJRIEPS 43 janvier 2018}

conception dominante de sport d'équipe. Enfin, 10\% des enseignants débutent leur réponse en signalant que le football est un sport de contact à connotation masculine. Au-delà de la première unité de sens renvoyant à la conception dominante du répondant, d'autres unités de sens venant dans la définition du football mettent en avant des conceptions secondaires chez la plupart des enseignants. Cette présence de conceptions dominantes et de conceptions secondaires corrobore un certain nombre de travaux mettant en avant l'existence de réseaux conceptuels plus ou moins homogènes chez les enseignants d'EPS au regard des APSA support de l'enseignement (Calmettes, Carnus, 2008).

Ces premiers résultats mettent également en avant que la conception dominante la plus fréquente chez les enseignants d'EPS tunisiens interrogés est celle qui renvoie au plus près de la logique interne du football et de son positionnement dans la classification des APSA. Nous pensons que l'influence des prescriptions institutionnelles véhiculées par les institutions de formation n'est pas étrangère à ce constat. En même temps, la plupart des auteurs majeurs en sports-collectifs s'appuient sur une définition articulant confrontation et collaboration. Ainsi par exemple pour Déléris « l'ensemble des sports collectifs se présente comme un jeu de duel entre deux collectifs médié par un engin, se déroulant dans un espace défini, duel qui génère tout un réseau d'interactions entre équipes et joueurs » (Déléris, 2005).

3. 1. 2. Que pensez-vous du football scolaire?

Nous avons utilisé la même méthode que pour la question précédente pour l'analyse des réponses à cette question. Ainsi, se dégagent chez les répondants plusieurs conceptions dominantes du football scolaire (graphique 2). 


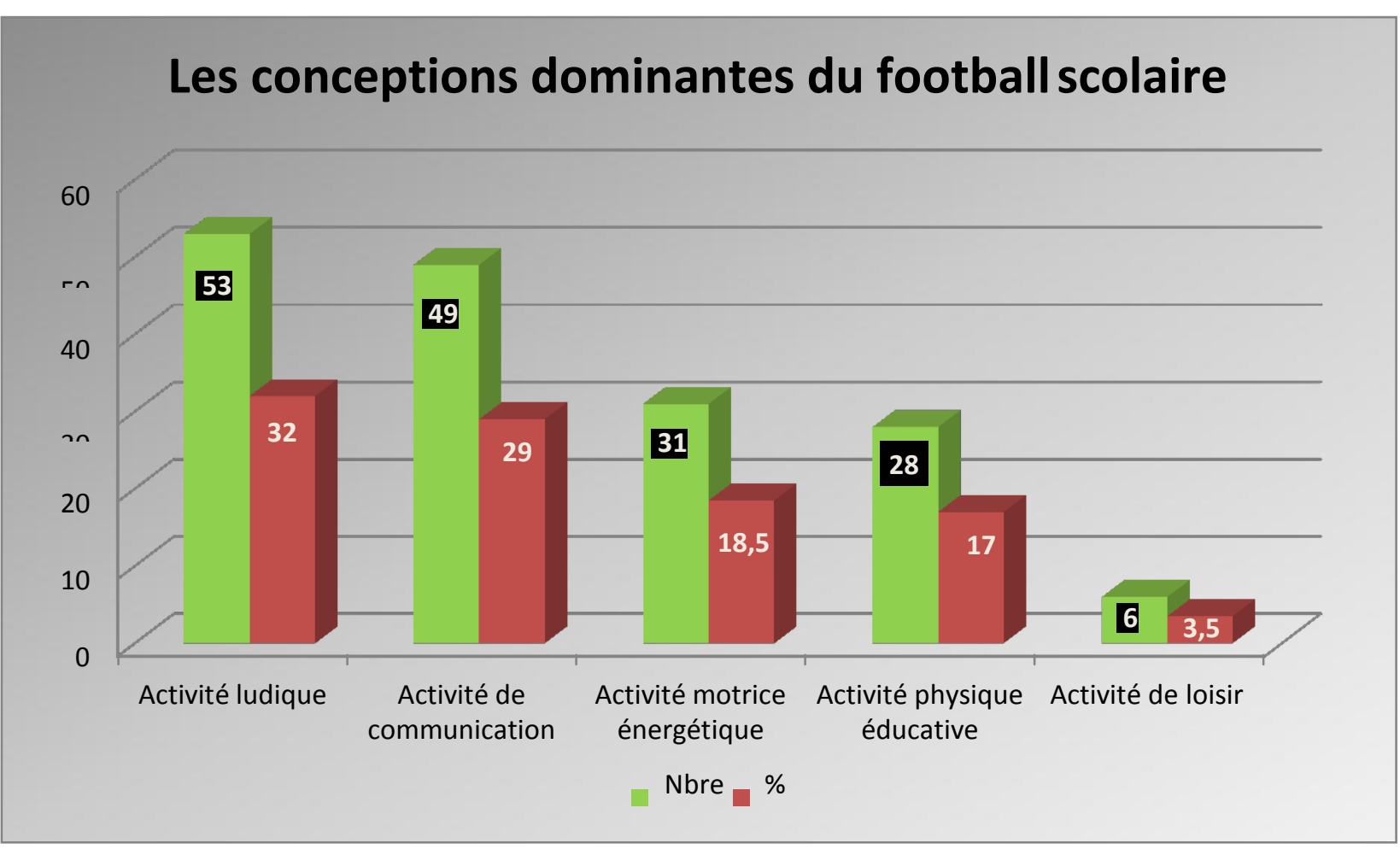

Graphique 2 . Les conceptions dominantes du football scolaire.

L'analyse qualitative et quantitative des réponses à cette question révèle des tendances différentes au regard de la question précédente. 32\% des réponses priorisent en premier lieu le caractère ludique du football scolaire que l'on peut rapprocher des $3,5 \%$ qui le considèrent comme une activité de loisir. Dans un autre registre de conceptions, $29 \%$ des réponses mettent en avant l'idée de communication entre les élèves à travers la pratique. Toutefois, les notions d'opposition et/ou de coopération que nous avions relevées pour la question précédente ne sont pas ici mentionnées. Une autre conception dominante apparaît dans $18,5 \%$ des réponses, celle d'une activité motrice de dépense énergétique. Enfin, pour $17 \%$ des répondants, le football en milieu scolaire est considéré comme une activité physique éducative développant des conduites motrices coordonnées, efficaces et sécuritaires. Développement de conduites motrices, coordination, efficacité, sécurité et éducation semblent liés dans les réponses relevant de cette catégorie. II sera utile pour la suite de notre travail d'approfondir cette conception dans des entretiens ${ }^{9}$.

Ainsi, l'analyse des réponses à cette deuxième question révèle l'influence sousjacente de conceptions plus génériques relatives à l'EPS apparemment réparties

\footnotetext{
${ }^{9}$ Notamment auprès des quatre enseignants d'EPS associés à notre recherche.
} 


\section{eJRIEPS 43 janvier 2018}

autour de quatre tendances : ludique, énergétique, éducative des conduites motrices et communicationnelle. II est intéressant de constater que le vocabulaire utilisé pour répondre à cette question est différent de celui relevé dans la question précédente et que la dimension culturelle de l'APSA n'apparaît pas dans les premiers items des réponses à cette question. Elle semble s'effacer au profit de conceptions plus fortement influencées par l'institution scolaire. Par ailleurs, le test de chi2 ne met en avant aucune liaison significative entre la conception dominante, le sexe, l'ancienneté ou l'expertise.

Le rapprochement de ces premiers résultats rend compte d'un processus de transposition didactique interne à l'œuvre au niveau des conceptions des enseignants mettant en exergue des écarts entre les conceptions du football fédéral et celles du football scolaire. II pointe aussi des tensions potentielles notamment entre plaisir et effort qui figurent au sein d'un déjà-là conceptuel singulier chez chaque enseignant. Qu'en sera-t-il des intentions didactiques poursuivies par les enseignants?

\section{2. Le déjà-là intentionnel}

Nous analysons ici les réponses à deux questions complémentaires : dans votre enseignement, quels sont vos centres d'intérêt ? (1) Quels sont les objectifs les plus importants pour vous? (2) Cette deuxième question se subdivise en trois champs de réponses : sur le plan technique, sur le plan tactique et en ce qui concerne les valeurs - autrement dit sur le plan éthique. La première question de nature fermée hiérarchise la fréquence des différents registres d'intentions et dissocie le déjà-là intentionnel spécifique (Loizon, 2004) ayant trait aux intentions didactiques (techniques et tactiques) du déjà-là intentionnel général (Loizon, 2004) portant sur des intentions éducatives à plus long terme (éthiques, sécuritaires, méthodologiques et sociales). La deuxième question, plus ouverte, cherche à inventorier qualitativement un ensemble d'enjeux de savoirs techniques, tactiques et éthiques visés par les enseignants lors de l'enseignement du football en EPS.

\section{2. 1. Les centres d'intérêt et priorités intentionnelles}

Pour cette première question, trois catégories de réponses sont proposées : techniques, tactiques, éducatifs avec pour chacune quatre choix possibles : toujours, souvent, rarement, jamais. Ainsi, se dégagent les priorités en matière d'intentions qui sont présentées dans le graphique 3 . 

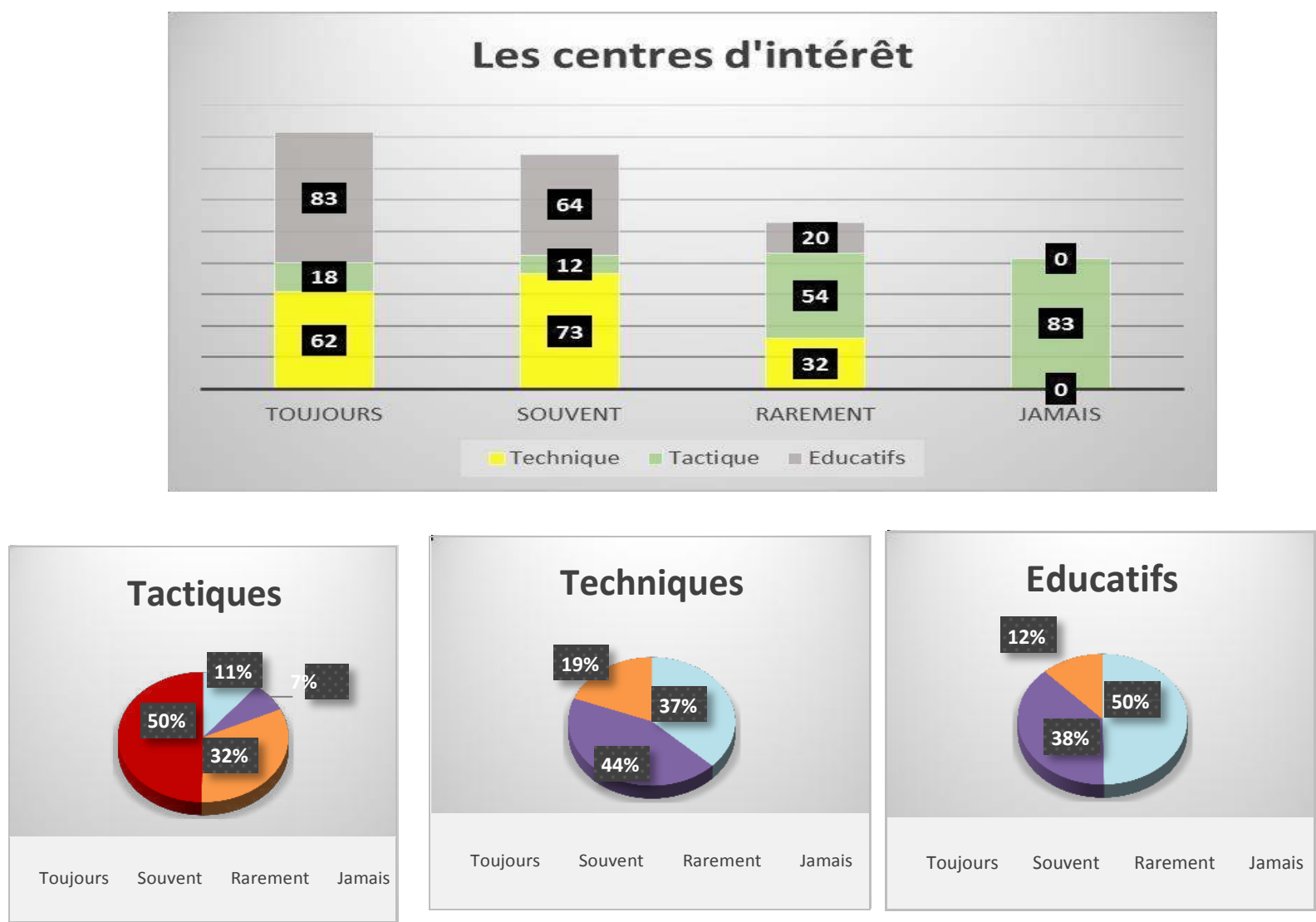

Graphique 3. Les centres d'intérêt et priorités intentionnelles des enseignants d'EPS.

Une première lecture des réponses à cette question met en avant une absence $(50 \%)$ ou une rareté $(32 \%)$ d'intentions de nature tactique chez les enseignants interrogés. Seuls $11 \%$ (toujours) et $7 \%$ (souvent) déclarent en poursuivre. A contrario, les intentions techniques (81\%) et éducatives (88\%) sont au cœur des préoccupations pour la grande majorité d'entre eux. Ces résultats apparaissent en décalage au regard des réponses relatives aux conceptions du football scolaire où seulement $17 \%$ des enseignants interrogés avaient mentionné la dimension éducative de l'activité en lien avec le développement de conduites motrices efficaces et coordonnées ${ }^{10}$.

3. 2. 2. Quels sont les objectifs les plus importants pour vous ?

Cette deuxième question relative au déjà-là intentionnel, de nature ouverte, invite les répondants à développer une réponse singulière quant à la nature des savoirs visés dansles trois catégories proposées : technique, tactique et éthique. Pour traiter cette

\footnotetext{
${ }^{10}$ Que l'on peut rapprocher des apprentissages techniques
} 


\section{eJRIEPS 43 janvier 2018}

question, nous avons procédé à une analyse catégorielle et effectué un rapprochement sémantique des unités de sens contenus dans les réponses. Chaque unité de sens renvoie à un savoir technique, tactique ou éthique. Parfois, plusieurs savoirs apparaissent dans une même réponse. Les graphiques 4, 5 et 6 visualisent l'ensemble des réponses.

- Sur le plan technique

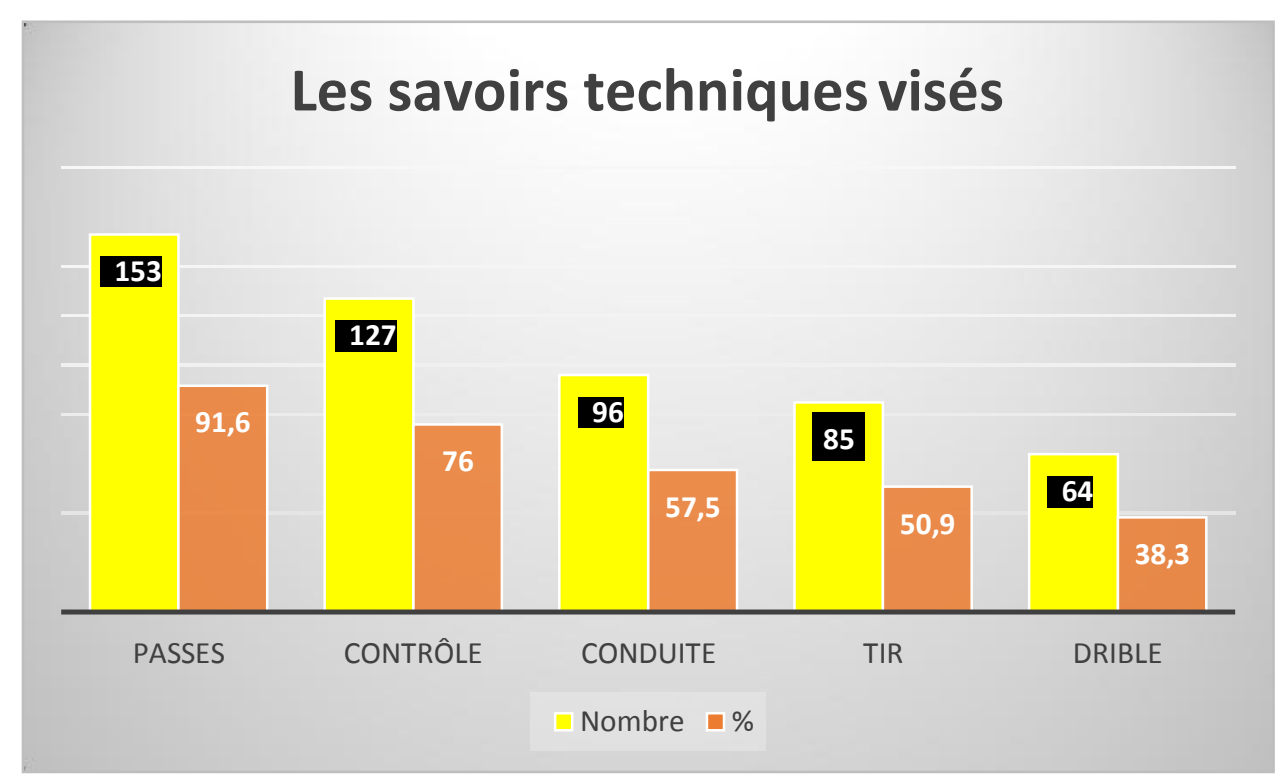

Graphique 4. Les savoirs techniques visés.

Les réponses à cette question permettent d'inventorier les éléments techniques de base sur lesquels les enseignants déclarent se focaliser. En premier lieu, on peut lire que près de $92 \%$ d'entre eux visent la construction de la passe, suivi du contrôle (76\%) et de la conduite de balle $(57,5 \%)$. Le tir n'est cité que par $51 \%$ et le drible mentionné par $38 \%$ des professeurs apparaît comme le savoir technique le moins recherché. Les deux techniques dominantes (passes et contrôle) sont en réalité complémentaires et particulièrement problématique en football du fait que la maîtrise du ballon qui se fait avec le pied nécessite un apprentissage technique spécifique qui rompt avec la motricité usuelle. Nous remarquons qu'aucun enseignant n'évoque l'apprentissage de techniques spécifiques au gardien de but dont le rôle est essentiel dans la pratique de haut-niveau mais qui en milieu scolaire n'apparaît pas comme un aspect à privilégier auprès des élèves. Ces choix techniques apparaissent ainsi en continuité avec certaines conceptions du football scolaire mettant en avant la 


\section{eJRIEPS 43 janvier 2018}

communication et le développement de conduite motrice coordonnée. Nous pointons aussi quelques décalages potentiels qu'il nous faudra approfondir entre l'importance accordée à la dimension ludique et à l'efficacité des conduites motrices au vu du score relatif obtenu par le tir.

- Sur le plan tactique

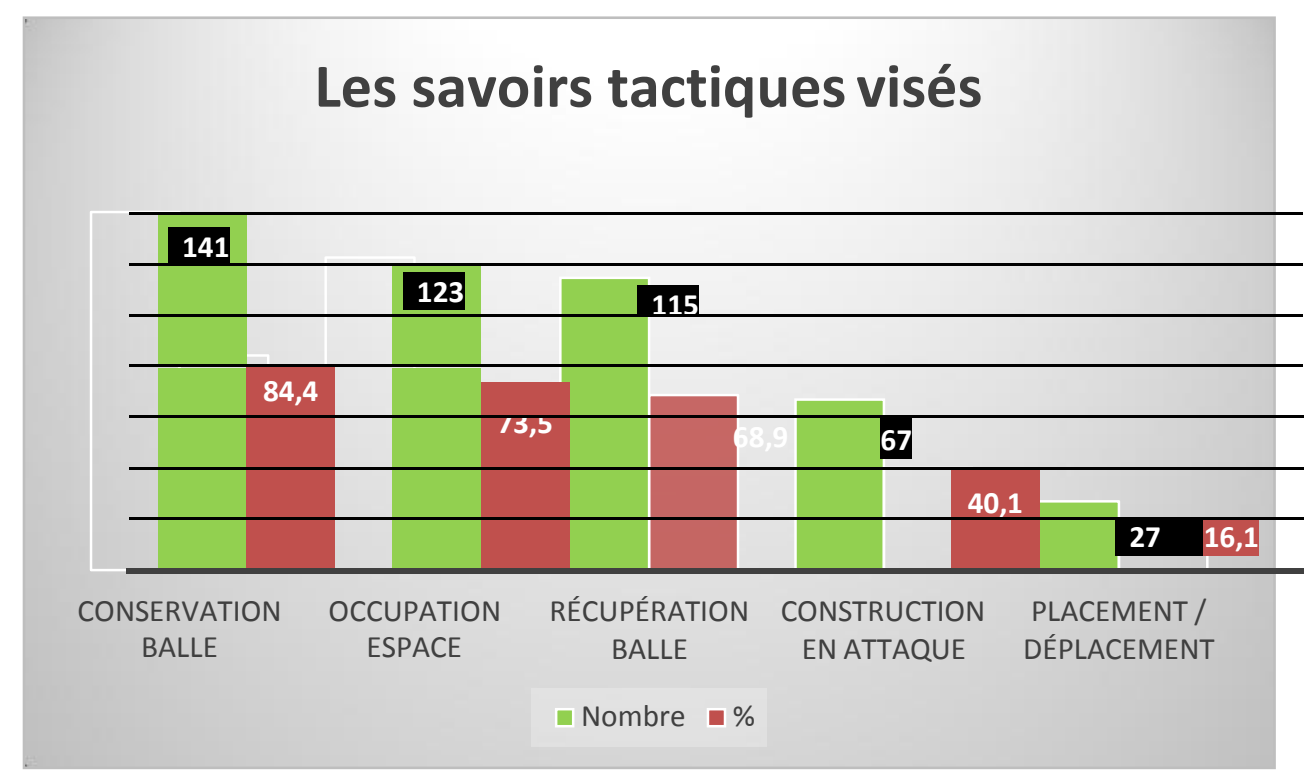

Graphique 5. Les savoirs tactiques visés.

Ce graphique affiche en nombre et en pourcentage, les objectifs tactiques les plus importants pour les enseignants. Alors qu'à la question précédente portant sur les centre d'intérêt nous avions relevé l'absence ou la rareté d'intentions tactiques pour la moitié des professeurs d'EPS interrogés (graphique 3), il est troublant de constater que 141 soit $84,4 \%$ d'entre eux citent la conservation de la balle Arrive en deuxième, l'occupation de l'espace plus particulièrement en attaque qu'en défense $(73,5 \%)$ suivi par la récupération de la balle $(68,9 \%)$. La construction en attaque est mentionnée par $40,1 \%$ d'entre eux. Enfin, il est intéressant de noter que la question du déplacement et du replacement est citée par 27 d'entre eux soit 16,1\%. Que révèlent ces réponses apparemment contradictoires relatives aux intentions tactiques ? En effet, bien que jugés importants par les enseignants, ces objectifs tactiques ne font pas partie des priorités intentionnelles de la moitié d'entre eux. S'agit-il de concessions ou de renoncements ? Et quelle en est la part conscientisée ? Là encore, cette étude exploratoire nous ouvre des pistes prometteuses pour interviewer les enseignants. 


\section{eJRIEPS 43 janvier 2018}

A travers ces réponses, nous constatons que les savoirs tactiques évoqués, en lien avec la logique des activités d'opposition et de coopération et la gestion d'un rapport de force, sont davantage focalisés sur l'attaque que sur la défense.

- En ce qui concerne les valeurs

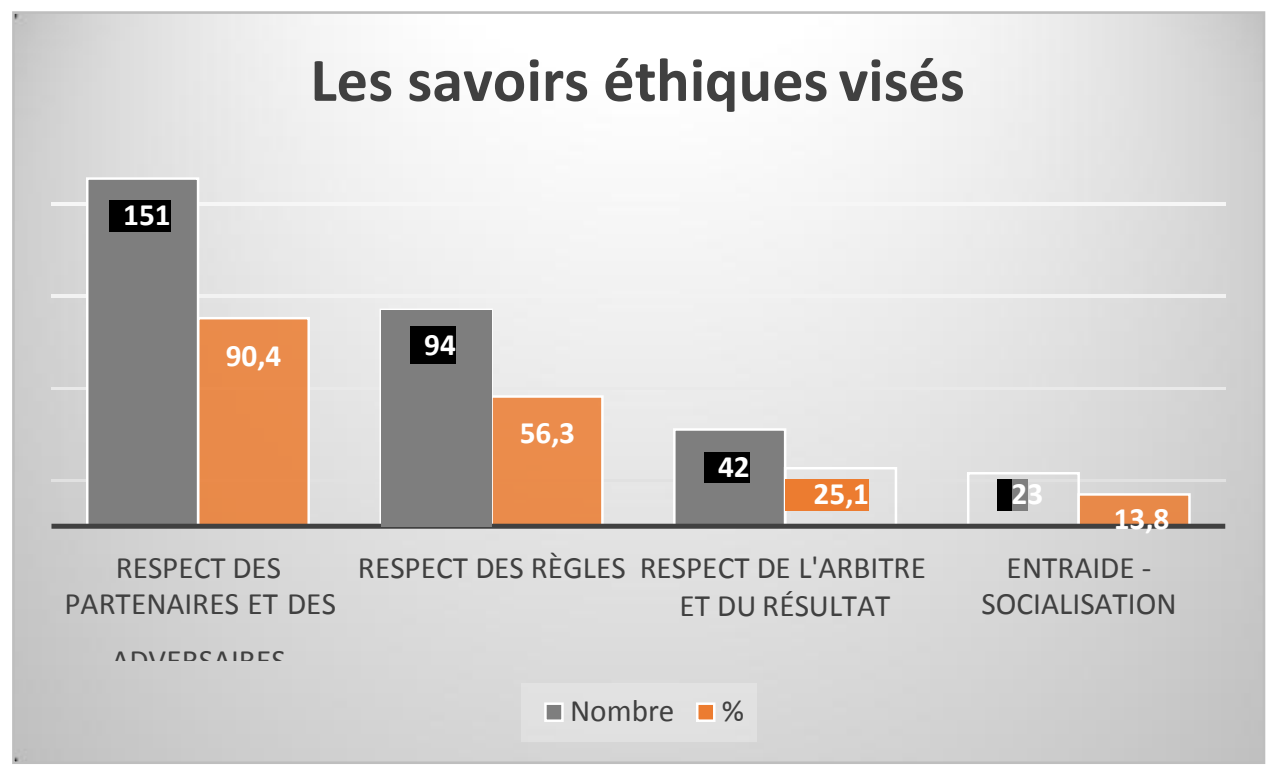

Graphique 6. Les savoirs éthiques visés.

Ce dernier point relatif au déjà-là intentionnel renvoie à un certain nombre de savoirs éthiques. Nous avons souhaité l'isoler dans la mesure où une des options de l'ingénierie que nous allons proposer aux enseignants associés consiste à composer des équipes homogènes entre-elles et mixtes et hétérogènes en leur sein. Compte tenu du caractère genré masculin de l'activité football nous avions besoin de repérer si certains d'entre-eux faisaient de la mixité et de l'acceptation des différences un objectif prioritaire. II s'avère que pour 151 d'entre-eux $(90,4 \%)$ le respect de l'adversaire et du partenaire est mis en avant suivi d'assez loin par le respect des règles $(56,3 \%)$ et celui de l'arbitre et du résultat $(25,1 \%)$. La dernière catégorie que nous avons repérée est relative aux notions d'entraide et de socialisation. Elle obtient le score modeste de 13,8\%. La question de la mixité n'est jamais évoquée.

Entre visées techniques, tactiques et éthiques, la trame intentionnelle des enseignants d'EPS s'organise au sein d'un déjà-là singulier au sein duquel émergent les savoirs à enseigner. Tout comme pour les conceptions, cet ensemble contribue à la compréhension du processus transpositif à l'œuvre au cours de l'enseignement du 


\section{eJRIEPS 43 janvier 2018}

football en EPS. Là encore, la recherche de liaisons significatives entre ces trames intentionnelles et le sexe, l'ancienneté ou l'expertise des enseignants s'est avérée improductive ${ }^{11}$. Qu'en sera-t-il des pratiques déclarées?

\section{3. Le déjà-la expérientiel}

Dans ce troisième et dernier volet du déjà-là décisionnel nous analyserons ici trois questions quinous intéressent particulièrement compte tenu des options de notre ingénierie. La première cherche à faire émerger les principales variables didactiques manipulées par les enseignants pour faciliter l'accès aux savoirs à l'élève. La seconde se focalise sur le type de situation qu'ils mettent en place pour évaluer les acquisitions des élèves. La troisième cible les différentes formes de groupement utilisées par les enseignants lors des séances.

3. 3. 1. Les principales variables didactiques déclarées par les enseignants

Pour Brousseau, « la situation simule un milieu qui détient des secrets que l'on peut lui arracher en les déduisant d'informations adéquates que l'on tire de lui » (Brousseau,1998, p. 309). Ce milieu se présente comme un ensemble de contraintes qui doivent garantir l'apprentissage de l'élève (Margolinas, 1993). Ces différentes contraintes sont qualifiées pour une grande part dans ce que Brousseau (1986) appelle les variables didactiques et qui représentent les « composantes de l'enseignement - apprentissage sur laquelle l'enseignant a pris la décision d'agir en adoptant pour chacune d'elles des modalités pratiques particulières " (Bru, 1991, p.95).

Pour accéder aux principales variables didactiques et leurs modalités manipulées par les enseignants nous avons posé la question suivante : " Pour faciliter l'apprentissage, que faites-vous varier le plus souvent? ". Chaque réponse renferme de 1 à 3 unités de sens renvoyant à une ou plusieurs variables didactiques. A la suite de cette question ouverte, nous avons demandé aux enseignants de préciser dans quel sens ils faisaient varier ces

paramètres et de justifier leur réponse. Le nombre de non-réponse à cette demande de précisions et de justifications est relativement élevé ${ }^{1212}$. Ceci souligne la difficulté déjà repérée à discourir sur ses pratiques (Carnus, 2002).

Seulement quatre variables sont ainsi identifiées : le nombre de touches de balle, le

\footnotetext{
${ }^{11}$ Ceci est à relativiser compte tenu du nombre d'enseignants interrogés.

${ }^{12}$ Près de $12 \%$ des enseignants interrogés n'ont ni précisé ni justifié leurs choix de variables.
} 


\section{eJRIEPS 43 janvier 2018}

nombre de défenseurs, l'espace de jeu et l'utilisation de joueurs « joker » en attaque (graphique 7).

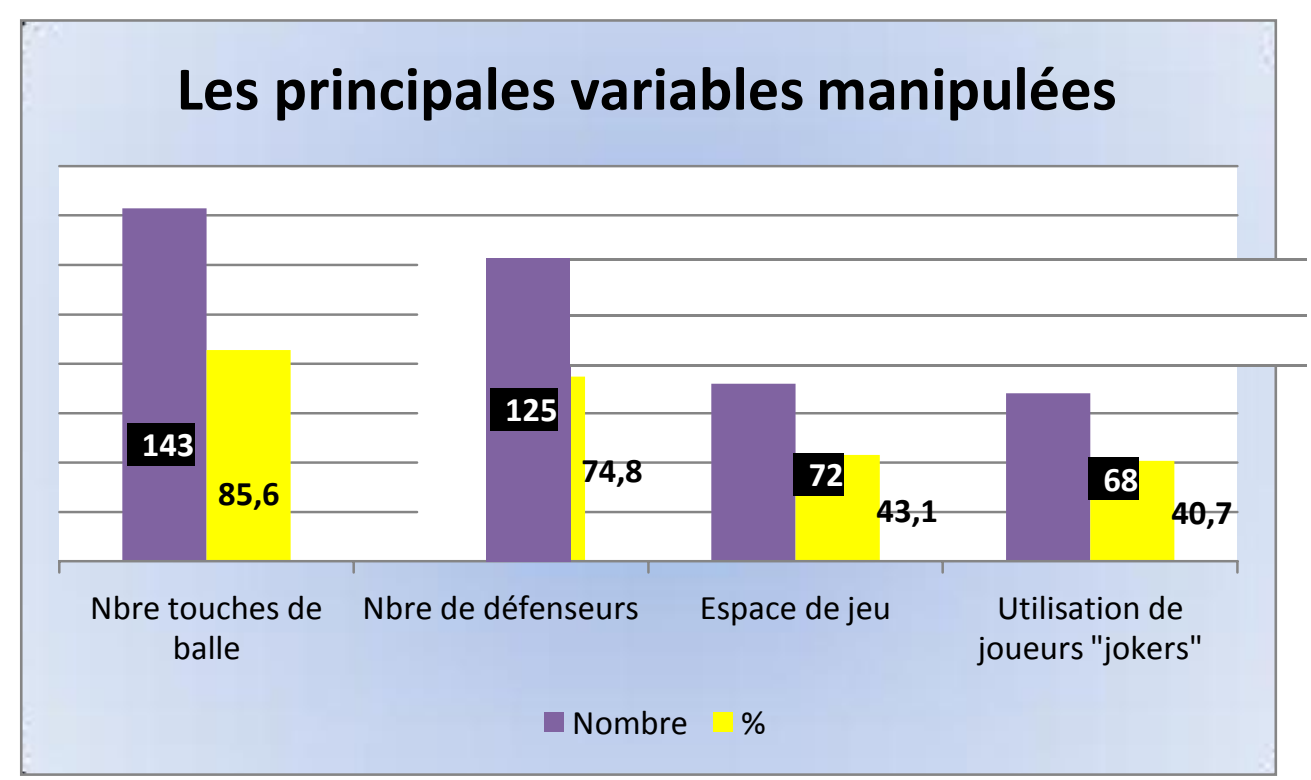

Graphique 7. Les principales variables manipulées par les enseignants.

Présent dans plus de $85 \%$ des réponses, le volume de jeu à travers l'augmentation du nombre de touches de balle arrive largement en tête et s'inscrit dans la continuité des intentions techniques relatives à la passe et tactiques visant la conservation de la balle. La variation du rapport de force en faveur d'un surnombre offensif que l'on installe par la diminution du nombre de défenseurs arrive en second avec un score de $74,8 \%$. De manière isolée ou combinée, on retrouve également cette stratégie d'exploitation d'un surnombre offensif pour $40,7 \%$ des répondants sous la forme d'utilisation de joueurs

« jokers » en attaque. Ces aménagements rendent compte d'une certaine cohérence avec les intentions tactiques ciblées sur l'organisation en attaque (graphique 5). La dernière variable citée est relative à l'espace de jeu et la modalité retenue pour la grande majorité des répondants est celle d'un agrandissement. Or plus l'espace de jeu augmente et plus la gestion collective de son occupation devient problématique. En même temps les ressources énergétiques des élèves sont davantage sollicitées. Entre l'intention tactique portant sur l'occupation collective de l'espace déclarée par plus de $73 \%$ des enseignants (graphique 5) et une conception énergétique de l'activité énoncée par 18\% d'entre eux (graphique 2), l'augmentation de l'espace de jeu que $43 \%$ des répondants déclarent mettre en œuvre pour faciliter l'apprentissage 


\section{eJRIEPS 43 janvier 2018}

semble relever d'une décision en tension entre un déjà-là intentionnel et un déjà-là conceptuel. Ce constat corrobore l'idée de tensions et parfois de contradictions au cœur du déjà-là décisionnel de l'enseignant d'EPS (Carnus, 2002).

3. 3. 2. Les situations d'évaluation des acquisitions des élèves

A la question "Quelles situations choisissez-vous comme situations d'évaluation ? " nous avons proposé un positionnement autour de trois types de réponse au regard de notre connaissance de l'enseignement usuel du football en milieu scolaire ${ }^{13}:$ des circuits techniques ? Des situations d'opposition décontextualisées de type analytique ? Des situations d'opposition contextualisées de type global ? Le graphique 8 présente la répartition des réponses à cette question.

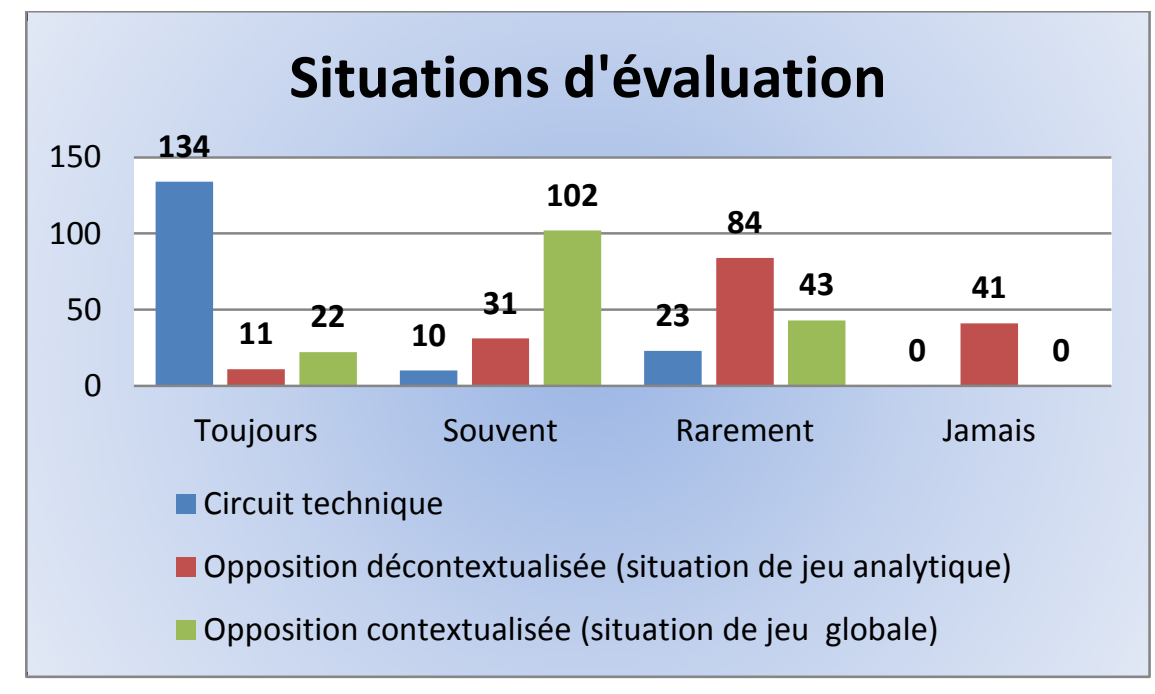

Graphique 8. Les situations d'évaluation.

Sur les 167 répondants, 134 soit plus de $80 \%$ déclarent mettre en place des circuits techniques pour évaluer les acquisitions de leurs élèves. Cette réponse s'inscrit dans la continuité des intentions techniques massivement investies par les enseignants d'EPS tunisiens interrogés (graphique 3). II est toutefois intéressant de constater qu'en même temps $61 \%$ d'entre eux (102) déclarent également mettre souvent en place à des situations d'opposition contextualisées sous forme de jeu global. Ces réponses confirment ce que nous avons maintes fois remarqué au cours de nos observations de pratiques ordinaires à savoir que les enseignants d'EPS ont recours à plusieurs types de situations au cours d'un même cycle pour évaluer de manière dissociée des savoirs techniques et des savoirs tactiques. Rares sont ceux qui

\footnotetext{
${ }^{13}$ Cette connaissance provient notamment des nombreuses visites d'enseignants débutants que nous effectuons dans le cadre de notre activité professionnelle.
} 


\section{eJRIEPS 43 janvier 2018}

évaluent leurs élèves dans des situations d'opposition décontextualisée qui favorisent l'apprentissage de gestes tactiques (notamment les situations de surnombre offensif).

Ainsi, nous dégageons une tendance très largement majoritaire qui met le circuit technique au cœur de l'évaluation qui comme l'a montré Pédèches en volley-ball « pilote le contrat didactique à l'insu de l'enseignant " (Pédèches, Carnus, 2013). En effet, le choix des modalités d'évaluation dépend des attentes plus ou moins conscientisées de l'enseignant. Le recours à différentes situations pointe à quel point " évaluer fait problème " (Hameline, 1982). En football l'évaluation présente encore plus de difficultés du fait des dimensions individuelles, collectives, techniques et tactiques de l'activité. Même si tous les élèves ne jouent pas en même temps, comment évaluer simultanément plusieurs joueurs dans un match d'environ 10 minutes ? Le choix d'un circuit technique dans le dispositif d'évaluation traduit-il un compromis permettant de garantir une forme d'équité ou une solution palliative au manque d'expertise de la plupart des enseignants qui ne sont pas spécialistes de l'activité ? S'agit-il d'une concession ou d'un renoncement?

\section{3. 3. Les différentes formes de groupement des élèves}

Pour accéder aux modalités de cette dernière variable que nous présentons ici nous avons demandé aux enseignants : "Au cours de la séance optez-vous pour un travail ? » en proposant trois types de réponses : travail individuel (1) ; travail par petits groupes unisexes (2) ; travail par petits groupes mixtes (3). Les formes de groupement mixtes - notamment dans les situations d'opposition contextualisées font partie des variables de commande de notre ingénierie. Au-delà d'un état des lieux en matière de forme de groupement majoritairement utilisées par les enseignants d'EPS tunisien en football, notre intention est de repérer d'éventuelles " zones d'achoppement " de nature à perturber l'habitus professionnel des enseignants associés à la recherche. Le graphique 9 synthétise et représente l'ensemble des réponses. 


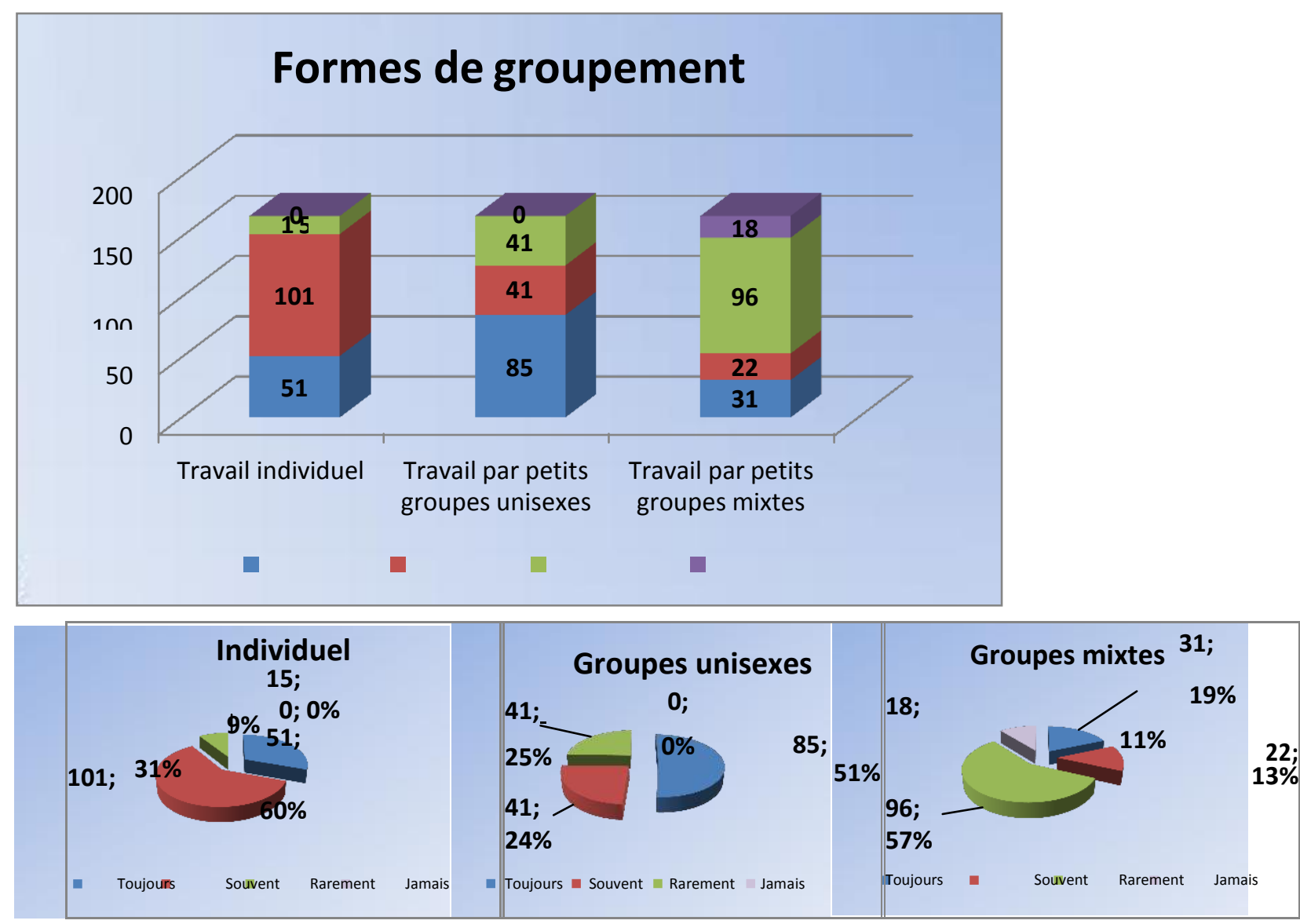

Graphique 9. Les formes de groupements utilisés.

Les réponses à cette dernière question sont sans appel. Les formes de travail individuel et par petit groupe unisexes sont combinés la plupart du temps au cours des séances et la mixité ne fait pas partie du paysage didactique en football pour $68 \%$ des enseignants d'EPS tunisien interrogés qu'ils soient hommes ou femmes, débutants ou expérimentés, spécialistes ou non spécialistes. $51 \%$ ont toujours recours aux groupes démixés et ce qui frappe c'est que $91 \%$ déclarent avoir toujours ou souvent recours à un travail individuel. Certes, cette modalité est de nature à favoriser la construction ou le renforcement de certains savoirs techniques attendus et par là même gérer l'hétérogénéité des publics scolaires. En même temps, elle questionne le sens et l'essence de l'activité. II sera intéressant d'approfondir cette question avec nos quatre collaborateurs au cours d'entretien. II aurait été pertinent d'inviter les enseignants à préciser et à justifier ce choix.

\section{Conclusion}

Dans les limites de validité locale et contextuelle (Van Der Maren, 1995), l'intérêt de 


\section{eJRIEPS 43 janvier 2018}

ce questionnaire est triple : celui de permettre l'émergence d'un déjà-là en tension chez un nombre relativement important d'enseignant d'EPS tunisiens ; celui de positionner les quatre enseignants qui ont accepté de collaborer à notre recherche ; celui de mesurer la proximité et la distance entre certains options de l'ingénierie qua nous souhaitons mettre en place pour observer les pratiques et les pratiques usuelles. Cet article s'est davantage focalisé sur le premier aspect.

En effet, cette étude exploratoire met en évidence la multiplicité des réseaux conceptuels, intentionnels et expérientiels au cœur du processus décisionnel de l'enseignant d'EPS. Entre sphère publique et privée, influences culturelles, institutionnelles et personnelles, les enjeux de savoir circulent, s'élaborent et émergent selon une logique singulière difficilement accessible. Ces analyses révèlent et corroborent un certain nombre de tensions au sein d'un déjà-là agissant de manière constante et latente en amont de la décision enseignant (Carnus, 2002) comme filtre de l'action didactique (Loizon, 2004). A travers ce questionnaire nous avons pu identifier certaines de ces tensions dans l'enseignement du football à travers la mise à jour de contradictions dans les conceptions fédérales et scolaires qui donnent du sens aux actions et les intentions qui les finalisent à plus ou moins long terme. Au cœur du déjà-là intentionnel se trouvent les savoirs à enseigner, produits sans cesse remaniés d'une épistémologie personnelle et professionnelle (Carnus, 2003)

Cette étude confirme également que c'est sur leurs pratiques que les enseignants ont le plus de mal à s'exprimer. Le nombre de non-réponse à certaines questions nous paraît significatif de la difficulté qu'ont les enseignants à mettre en mots leurs gestes professionnels quotidiens, qu'ils soient débutants ou expérimentés, spécialistes ou non spécialistes. Néanmoins l'analyse didactique clinique des pratiques déclarées par les enseignants d'EPS Tunisien prépare et outille notre regard et nous ouvre des perspectives heuristiques pour la compréhension de ce qui s'enseigne réellement en football en milieu scolaire en Tunisie. Ainsi, en prenant en compte le sujet dans ses dimensions psychologique et sociale (Beillerot, BlanchardLaville, Mosconi, 1996), l'approche clinique en didactique apporte une autre intelligibilité à la complexité du processus transpositif à l'œuvre dans le fonctionnement didactique (Carnus, 2010).

Notre recherche se poursuit. Des entretiens avec nos quatre collaborateurs 


\section{eJRIEPS 43 janvier 2018}

permettront de dessiner plus précisément les contours de leur déjà-là décisionnel singulier et de repérer avec une plus grande acuité les tensions structurelles qui les animent avant d'aller les observer, in situ et au cas par cas au cours d'un cycle de football. Quels seront les savoirs réellement enseignés au moment de l'épreuve de l'interaction?

\section{Bibliographie}

Artigue, M., Ingénierie didactique. Recherches en Didactique des Mathématiques, 9/3,1990, pp. 283-307.

Amade-Escot, C. \& Léziart, Y., Contribution à l'étude de la diffusion de propositions d'ingénierie didactique auprès de praticiens. Analyse de cas d'enseignants d'éducation physique et sportive volontaires. Rapport scientifique. Recherche INRP ; N³0506, 1996.

Bardin, L. (1998). L'analyse de contenu, 9e édition. Paris : PUF.

Beillerot, J., Blanchard-Laville, C., Mosconi, N. (1996). Pour une clinique du rapport au savoir. L'Harmattan, Paris.

Brousseau, G. (1986). La relation didactique : le milieu. Actes de la IVème Ecole d'Eté de didactique des mathématiques, pp. 54-68, IREM, Paris 7.

Brousseau, G. (1998). Théorie des Situations Didactiques. Grenoble : La pensée sauvage.

Bru, M. (1991). Les variations didactiques dans l'organisation des conditions d'apprentissage. EUS, Toulouse.

Calmettes, B. \& Carnus, M.-F. (2008). Etude des évolutions du rapport au savoir des étudiants de première année IUFM et des professeurs stagiaires en physique- chimie et en EPS, in M.-F. Carnus, C. Garcia-Debanc et A. Terrisse (dir). Analyse de pratiques des enseignants débutants, approche didactique, La pensée sauvage, Grenoble, 17-34.

Carnus, M.-F. (2001). Analyse didactique du processus décisionnel de l'enseignant d'EPS en gymnastique. Une étude de cas croisés. Thèse de doctorat, Université Paul Sabatier.

Carnus, M.-F. (2002). Croyances, conceptions, intentions et pratiques effectives dans l'enseignement de la gymnastique : Le cas de l'ATR et du repérage. In J.-F. 


\section{eJRIEPS 43 janvier 2018}

Robin et A. Durny (dir), Travaux d'actualité en activités gymniques et acrobatiques (p. 160-164). Dossiers EPS, 57, Paris.

Carnus, M.-F. (2003). Analyse didactique du processus décisionnel de l'enseignant d'EPS en gymnastique : une étude de cas croisés. In C. Amade-Escot, Didactique de l'éducation physique, état des recherches. Paris : Éditions EP\&S, 193-224

Carnus, M.-F. (2009). La décision de l'enseignant en didactique clinique. Etudes de cas en Education Physique et Sportive. In A. Terrisse et MF. Carnus (dir), Didactique clinique de l'EPS, Quels enjeux de savoirs ?, Bruxelles, De Boëck, 63- 81.

Carnus, M.-F. (2010). L'inscription clinique en didactique de l'éducation physique et sportive. Revue Cliopsy $n^{\circ} 4,73-88$.

Carnus, M-F. \& Terrisse A. (2013). Didactique clinique de l'EPS. Le sujet enseignant en question. Revue EPS (eds.). Collection Recherche et Formation. Paris.

Cazola, G., \& Farhi, A. (1998). Football : exigences physiques et physiologiques actuelles. Revue EPS, 273,60-66.

Chevallard, Y. (1985). La transposition didactique. La pensée sauvage, Grenoble.

Chevallard, Y. (1992). Concepts fondamentaux de la didactique : perspectives apportées par une approche anthropologique, Recherches en didactique des Mathématiques, 12/1. La pensée Sauvage, Grenoble, 73-112.

Deleris, C. (2005). Enseigner les sports collectifs en milieu scolaire (collèges, lycées) : compétences, contenus d'enseignement, évaluation : Actio.

Giordan, A. \& De Vecchi, G. (1987). Les origines du savoir. Des conceptions des apprenants aux concepts scientifiques, Neuchâtel-Paris, Delachaux et Niestlé.

Hameline, D. (1982). Les objectifs pédagogiques en formation initiale et en formation

Heuser, F. (2009). Du savoir enseigné et évalué à la référence de l'enseignant d'EPS : étude didactique clinique en karaté, Thèse de doctorat, Université Paul Sabatier.

Loizon, D. (2004). Analyse des pratiques d'enseignement du judo : identification du savoir transmis à travers les variables didactiques utilisées par les enseignants en club et en EPS. Thèse de doctorat, Université Paul Sabatier, Toulouse. 


\section{eJRIEPS 43 janvier 2018}

Loizon, D. (2009). " Les filtres personnels dans l'action didactique. Etude de cas en EPS ", in Terrisse (A.) et Carnus (M.-F.). Didactique clinique de l'éducation physique et sportive (EPS). Quels enjeux de savoirs ? Bruxelles, Édition De Boeck, 83-99.

Loizon, D. \& Carnus M.-F. (2012). Le déjà-là décisionnel chez les enseignants novices en EPS. Un outil pour comprendre la réalité de leurs enseignements, in Carlier (G.), Borges (C.), Clerx (M.) et Delens (C.), Identité professionnelle en éducation physique. Parcours des stagiaires et enseignants novices. Louvain-la-Neuve, Presses Universitaires de Louvain, 199-215.

Margolinas, C. (1993). De l'importance du vrai et du faux dans la classe de mathématiques, Grenoble, La Pensée Sauvage Éditions.

Pédèches, P. \& Carnus, M-F. (2013). Quand l'évaluation pilote le contrat didactique à l'insu de l'enseignant. Etude de cas en volley-ball. In MF Carnus \& A. Terrisse (dir.). Didactique clinique de l'EPS. Le sujet enseignant en question. Revue EPS (eds.). Collection Recherche et Formation. Paris. 71-80.

Portugais, J. (1999). Esquisse d'un modèle des intentions didactiques, Contribution à la didactique des mathématiques. In J. Brun, F. Conne, R. Floris et ML

Schubauer- Léoni Interactions didactiques, Méthodes d'étude du travail de l'enseignant. (pp. 57-88). Actes des secondes journées didactiques de la Fouly. Grenoble : La pensée sauvage.

Terrisse, A. (2000). Épistémologie de la recherche clinique en sports de combat. In A. Terrisse (sous la direction de), Recherches en Sports de combat et Arts Martiaux, État des lieux (pp. 95-108). Paris, Éditions revue EP\&S.

Terrisse, A., Carnus, M.-F., Loizon, D. (2010). La didactique clinique de l'EPS : perspectives pour la formation. In M. Musard, M. Loquet, G. Carlier (sous la direction de), Sciences de l'intervention en EPS et en sport. Résultats de recherches et fondements théoriques (pp. 137-158). Paris : Éditions EP\&S.

Touboul, A. (2010). Le poids de l'expérience et de l'expertise dans l'enseignement de la boxe savate française. Une étude en didactique clinique de l'EPS, mémoire de doctorat, Université Paul Sabatier.

Van Der Maren, J. M. (1995). Méthodes de recherche pour l'éducation. De Boeck, Bruxelles. 